\title{
Influência das Condições Ambientais e Ação Antrópica Sobre a Eficiência Produtiva Agropecuária em Minas Gerais
}

\author{
Samuel Alex Coelho Campos ${ }^{1}$, Alexandre Bragança Coelho ${ }^{2}$ e \\ Adriano Provezano Gomes ${ }^{3}$
}

Resumo: A produção agropecuária causa impactos sobre o meio ambiente e este também influencia a agropecuária. Apesar da importância do meio ambiente, a forma de produção atual é apontada como não sustentável e depreciativa. Neste sentido, buscou-se avaliar a importância dos recursos naturais sobre a produção agropecuária em Minas Gerais e das ações humanas de conservação do meio ambiente. Foi utilizada a Análise Envoltória de Dados e, posteriormente, a regressão quantílica, tendo como fonte de dados o Censo Agropecuário 2006 para os municípios mineiros. Os resultados demonstram a importância significativa das práticas agropecuárias sobre a produção agropecuária dos municípios. As variáveis "Plantio em Nível" e "Terraços" apresentaram a maior importância, sendo estas estatisticamente diferentes entre os quantis. Este resultado pode ser atribuído ao relevo mineiro e à concentração de municípios em regiões de relevo acidentado.

Palavras-chaves: Meio ambiente, eficiência, produção agropecuária, Análise Envoltória de Dados.

Abstract: Agricultural production has major effects on the environment; the environment, in turn, affects farming. Despite the importance of the environment, the form of current production is treated as unsustainable and derogatory. In this sense, we have evaluated the importance of this on agricultural production and human actions to conserve the environment. Data Envelopment Analysis and the quantile regression were used. We have used the 2006 Census of Agriculture as a source of data for the municipalities in Minas Gerais state. Results show the significant importance of human actions to conserve the environment on the agricultural production of municipalities and the variables "Plantio

\footnotetext{
1 Escola Superior de Agricultura "Luiz de Queiroz"/Universidade de São Paulo. E-mail: samuel.coelho@yahoo.com

2 Departamento de Economia Rural/Universidade Federal de Viçosa. E-mail: acoelho@ufv.br

3 Departamento de Economia/Universidade Federal de Viçosa. E-mail: apgomes@ufv.br
} 
em Nivel" (Planting Level) and "Terraços" (Terraces) have a major importance. This result can be attributed to the relief and the concentration of municipalities in regions of rugged terrain.

Key-words: Environment, efficiency, agricultural production, Data Envelopment Analysis.

Classificação JEL: D12, Q53.

\section{Introdução}

O agronegócio é tido como a soma de todas as operações ao longo da cadeia agroalimentar, compreendendo o setor fornecedor de insumos, a produção a nível primário, processamento e distribuição. A importância deste setor está ligada fundamentalmente, segundo Batalha e Scarpelli (2005), ao papel que seus produtos ocupam em qualquer grupo social, sendo que os alimentos, além de serem fundamentais para a vida, estão ligados a fatores sociológicos, antropológicos e psicológicos que conferem valores comportamentais e identidade cultural a uma população.

Considerando-se os aspectos econômicos do agronegócio, este tem grande importância para a economia brasileira, sendo um dos principais responsáveis pelo saldo positivo na balança de pagamentos do Brasil. As exportações do agronegócio brasileiro em 2008, segundo Brasil (2009), totalizaram US\$ 58,4 bilhões em produtos agrícolas, com crescimento médio anual de $20,43 \%$ entre 2000 e 2008, absorvendo quatro bilhões de dólares em investimentos diretos em 2008 (BACEN, 2008).

O agronegócio é de grande importância para o estado de Minas Gerais. Segundo dados do Cepea (2009), o estado representou $12 \%$ do agronegócio brasileiro em 2008, com crescimento de $26 \%$ na participação nacional em relação a 2001. Tal setor contribuiu para o PIB mineiro com R\$ 93.529 milhões em 2008. Dentre as atividades agropecuárias, merece destaque a produção de leite, em que o estado é o maior produtor nacional e que representou 51,8\% do PIB do agronegócio mineiro, ou $\mathrm{R} \$ 90,5$ bilhões em 2008 , segundo
Zuccolotto et al. (2009). Destaque também para a produção de batata-inglesa, responsável por 33\% da produção nacional (maior produtor nacional) e do feijão, em que Minas Gerais é o segundo produtor nacional, (17\% da produção nacional em 2008), entre outros (SIDRA, 2008).

Entretanto, a produção agropecuária enfrenta constantes ameaças, como a internacionalização com a abertura comercial, que expõe o produtor a produtos com preços artificialmente baixos, riscos de mercado e de produção, aumentos dos custos de produção e redução dos preços dos produtos agropecuários. Alvim e Oliveira Júnior (2005) destacam as mudanças nas vantagens competitivas, resultado das inovações tecnológicas e das práticas ecologicamente corretas. De tal forma, é necessário que produtores utilizem os recursos disponíveis para a produção de forma racional, produzindo o máximo possível dado o nível de recursos disponíveis. Cespedes e Leandro (2005) consideram, ainda, que uma produção eficiente contribui para o bem-estar da sociedade, embora estes autores desconsiderem a sustentabilidade ambiental.

Entretanto, a produção agropecuária é dependente de vários fatores externos ao controle das firmas, tendo importante influência sobre produção e produtividade agropecuárias: o meio físico, como clima, solo, pluviosidade etc. (AMARAL et al., 2004; MOREIRA, 2002). Outros autores, como Zilberman et al. (1999) e López (1997), acreditam na interdependência entre agropecuária e meio ambiente. As variáveis ambientais propiciam o ambiente físico ao desenvolvimento das atividades agropecuárias e a ação produtiva interfere no ambiente, que age sobre a agropecuária, 
formando um ciclo. Carneiro et al. (2005) destacam a importância da aptidão agrícola das terras sobre a produção e produtividade, bem como as variáveis geográficas passivas ${ }^{4}$ e ativas $^{5}$. A eficiência ou ineficiência da produção agropecuária é resultado não somente de fatores controláveis pelos produtores como terra, trabalho e capital, mas também das condições ambientais, como a fertilidade natural do solo e o clima que podem fazer com que produtores, submetidos a restrições ambientais, utilizem mais fatores e insumos como forma de compensar essas restrições, e não pela ineficiência.

A ação da agropecuária sobre o meio ambiente é tratada por Rodrigues (2005), Soares et al. (2005), Engström et al. (2007) e El Khalili (2009), que destacam a forma não sustentável da produção e os impactos ambientais, como emissão de gases do efeito estufa via queimadas e desmatamentos, assoreamento dos rios, desertificação, eutroficação e salinização dos solos. Estes autores dão enfoque aos impactos da agricultura e ações para sua minimização.

Desta forma, torna-se importante conhecer os efeitos das variáveis ambientais e das práticas agropecuárias adotadas pelos municípios sobre a ineficiência técnica. A princípio espera-se um aumento da produção dado o nível de utilização dos insumos (redução da ineficiência) com o aumento da utilização de práticas agropecuárias de conservação do meio ambiente pelos municípios, além de uma redução da ineficiência técnica daqueles municípios que possuam condições naturais favoráveis, uma vez que municípios que tenham solos férteis, por exemplo, demandariam um menor nível de utilização de fertilizantes para obter um mesmo nível produtivo que aqueles municípios com fertilidade natural inferior. Espera-se que a determinação da importância do meio ambiente sobre tal setor produtivo e das práticas agropecuárias contribua para a conscientização da importância do meio ambiente como

4 As variáveis passivas são estáticas ou incontroláveis, como clima, precipitação, temperatura, relevo, topografia etc.

5 As variáveis geográficas ativas podem ser alteradas ao longo do tempo por meio da tecnologia, como as características físicas e químicas dos solos. fator produtivo e das ações de preservação e/ou minimização dos impactos ambientais ocasionados pela atividade produtiva.

Este artigo está dividido em quatro seções, além desta. Na primeira, faz-se uma breve revisão teórica sobre o conceito de eficiência e métodos de estimação. Na segunda seção, é descrita a metodologia utilizada, dados utilizados e sua fonte. Na terceira seção, os resultados são apresentados e discutidos. Posteriormente, são feitas as conclusões finais, apontadas as limitações do estudo e sugestões para pesquisas futuras.

\section{Referencial teórico}

Uma firma é eficiente tecnicamente, na definição de Pareto, segundo Koopmans (1951), se quaisquer produtos não puderem ser aumentados, sem que, com isso, outro produto necessite ser reduzido, ou nenhum dos insumos utilizados pela firma possa ser reduzido sem que, com isso, a produção se reduza, ou qualquer outro insumo necessite ser aumentado para que o nível produtivo se mantenha. A eficiência técnica é definida por Pascual (2000) como a combinação ótima de insumos que permita a máxima produtividade dos produtos.

Tal nível de eficiência técnica é normalmente obtido por meio dos desvios frente à Fronteira de Produção, sendo proposta inicialmente por Debreu (1951). Na determinação das fronteiras de produção são comumente utilizados dois métodos. A fronteira de produção estocástica, obtida por Máxima Verossimilhança, impondo explicitamente uma forma funcional aos dados e acomodando ruídos. O segundo método, a Análise Envoltória de Dados (DEA), estima a fronteira por métodos de programação linear, não necessitando de imposição de uma forma explícita e acomodando vários produtos e insumos na estimação, embora ignore fatores não controláveis pelas firmas.

$\mathrm{Na}$ estimação das fronteiras de produção e dos níveis de eficiência das firmas, ambos os modelos podem ser empregados, não havendo um modelo preferencial, devendo ser utilizado aquele 
com melhor ajuste às pressuposições feitas e à disponibilidade e natureza dos dados. Assim, neste trabalho, optou-se pela "Análise Envoltória de Dados", pois ela se mostrou mais adequada, considerando-se o número de observações e o número de parâmetros a serem estimados e, também, por não necessitar de uma forma funcional explícita.

A formulação de problemas de medidas de eficiência como problemas de programação linear foi concebida pela primeira vez por Boles (1966), segundo Burgess e Wilson (1993). Entretanto, segundo Lins e Mesa (2000), foi com o empenho de Charnes, Cooper e Rhodes (1978) que os modelos DEA ganharam maior penetração a partir do modelo original CCR (sigla para Charnes, Cooper e Rhodes), que pressupõe retornos constantes à escala. Posteriormente, Banker, Charnes e Cooper (1984) propuseram uma modificação no modelo original, incorporando a pressuposição de retornos variáveis à escala, já que o modelo CRS-CCR pressupõe que todas as $\mathrm{DMUs}^{6}$ estão operando em escala ótima (COELLI, 1999). Posteriormente, o modelo foi modificado permitindo retornos não crescentes e não decrescentes à escala, estimação por modelo em dois estágios e multiestágio (buscando eliminar folgas do modelo), modelo aditivo, com fronteira invertida, modelo Free Disposal Hull, incorporando ao modelo variáveis não discricionárias e dummies, modelo de supereficiência e vários outros, buscando análises específicas e a adequação aos dados. $\mathrm{O}$ modelo de supereficiência foi desenvolvido inicialmente por Andersen e Petersen (1993) e permite ranquear as firmas eficientes.

A programação linear estima uma fronteira de produção convexa poliangular-linear, em que se calculam os desvios das firmas a esta fronteira de forma radial. O modelo tem como pressuposição a homogeneidade das DMUs, ou seja, estas

6 O termo DMU, Decision Making Unit, é utilizado na literatura sobre Análise Envoltória de Dados, já que tal metodologia não é aplicada apenas a firmas, mas às mais diversas instituições. O termo DMU refere-se a qualquer unidade tomadora de decisão que transforme insumos em produtos, podendo ser firmas, instituições, setores da economia ou regiões, como esse trabalho que estima a eficiência técnica dos municípios mineiros. realizam as mesmas tarefas com os mesmos objetivos sobre as mesmas condições de mercado, utilizando os mesmos insumos e gerando os mesmos produtos, em magnitudes diferentes (LINS e MOREIRA, 2000).

Atualmente, a Análise Envoltória de Dados tem sido largamente utilizada em diversas áreas, como em economia agrícola (GOMES, 1999; FERREIRA, 2002; GONÇALVES et al., 2008); em aplicações no universo bancário, como em Araújo e Carmona (2002); na educação, como em Moita (1995), Ruggiero e Vitaliano (1999); nas eleições, como Green et al. (1996) e Hashimoto (1997); na aviação, como em Fethi (2000); em P\&D, como em Lee e Park (2005); no transporte urbano, como Azambuja (2002); na indústria do aço, como em Ray e Kim (1995); em hospitais, como em Chilingerian (1995) etc.

As avaliações de eficiência podem, segundo Gomes e Baptista (2004), ser obtidas por meio de duas orientações - aquela que se fundamenta na variação dos insumos com a produção permanecendo constante, denominada orientação insumo; e aquela que coloca ênfase na variação do produto, mantendo os insumos constantes, denominada orientação produto. Neste trabalho, será utilizada a orientação produto, considerando que os insumos não são de grande mobilidade e os produtores rurais buscam a maximização da produção, utilizando o modelo de supereficiência, de forma a permitir melhor discriminação das DMUs (eficientes). Na escolha da orientação quanto aos retornos à escala, será utilizado o teste não paramétrico de duas amostras de Kolmogorov-Smirnov sugerido por Banker (1996).

\section{Metodologia}

Neste trabalho serão utilizados os modelos "tradicionais" propostos por Charnes, Cooper e Rhodes (1978) e Banker, Charnes e Cooper (1984) que estimam a eficiência sobre a pressuposição de retornos constantes (CRS/CCR) e variáveis (VRS/ $\mathrm{BCC}$ ), de forma a permitir interpretações quanto aos níveis de eficiência e reduções na utilização 
dos insumos. Na estimação da regressão quantílica será utilizado o modelo proposto por Andersen e Petersen (1993) de supereficiência, que permite a discriminação das firmas eficientes, uma vez que os escores dessas podem assumir valores entre 1 e infinito e, assim, permitem um melhor ajustamento da regressão quantílica.

\subsection{Análise envoltória de dados}

A Análise Envoltória de Dados consiste na estimação da fronteira de produção para as DMUs da amostra através de programação linear. A partir da fronteira, são estimados os escores de eficiência técnica para cada DMU por meio de movimentos radiais, sendo que as firmas eficientes encontram-se na fronteira de produção e as ineficientes, abaixo da fronteira.

Assim sendo, o modelo DEA com pressuposição de retornos constantes (CCR/CRS) e orientação produto consiste na solução do modelo abaixo, que deve ser solucionado para cada uma das DMUs:

Maximizar $_{\theta, \lambda} \phi$,

$$
\begin{gathered}
\text { s.a } \varphi \mathrm{y}_{\mathrm{i}}-\mathrm{Y} \lambda \leq 0, \\
-\mathrm{x}_{\mathrm{i}}+\mathrm{X} \lambda \leq 0 \\
-\lambda \leq 0
\end{gathered}
$$

em que $Y$ é uma matriz de $m$ produtos por $n$ DMUs; $X$ é uma matriz de insumos de ordem $\mathrm{k}$ insumos por $n$ DMUs; $y_{i}$ é o vetor de produtos, $x_{i}$ é o vetor de insumos, $\phi$ é um escalar e $\lambda$ é um vetor de pesos de ordem $n$ DMUs por 1 . A eficiência técnica é dada por $\theta=1 / \phi$. Para a estimação do modelo sobre retornos variáveis, deve-se adicionar a restrição $\mathrm{N1}^{\prime} \lambda=1$, em que N1 é um vetor $\mathrm{nx1}$ composto por uns.

A eficiência técnica orientada a produto, $\operatorname{TE}_{0}(x, y)$, satisfaz as seguintes propriedades (KUMBHAKAR e LOVELL, 2000):

(i) $\mathrm{TE}_{\mathrm{o}}(\mathrm{x}, \mathrm{y}) \leq 1$;

(ii) $\mathrm{TE}_{\mathrm{o}}(\mathrm{x}, \mathrm{y})=1 \Leftrightarrow \mathrm{y} \in \operatorname{Isoq} \mathrm{P}(\mathrm{x})$;

(iii) $\mathrm{TE}_{\mathrm{o}}(\mathrm{x}, \mathrm{y})$ é não decrescente em $\mathrm{y}$;

(iv) $\mathrm{TE}_{\mathrm{o}}(\mathrm{x}, \mathrm{y})$ é homogênea de grau 1 em $\mathrm{y}$;

(v) $\mathrm{TE}_{\mathrm{o}}(\mathrm{x}, \mathrm{y})$ é invariável com relação às unidades em que $x$ e y são medidas.
A propriedade (i) refere-se à homogeneidade; a propriedade (ii) refere-se a que uma firma eficiente está na isoquanta; a propriedade (iii) refere-se ao fato de que $\mathrm{TE}_{\mathrm{o}}(\mathrm{x}, \mathrm{y})$ não se reduz quando a produção de qualquer produto aumenta; a propriedade (iv) indica que uma mudança equiproporcional em todos os produtos resulta em uma mudança equivalente, de mesma direção, em $\mathrm{TE}_{\mathrm{o}}(\mathrm{x}, \mathrm{y})$; e pela propriedade (v), as unidades em que qualquer insumo e/ou produtos são medidos não afetam os escores de eficiência.

O modelo de supereficiência, proposto por Andersen e Petersen (1993), permite que os escores das firmas eficientes assumam valores entre 1 e infinito, sendo os escores das firmas ineficientes iguais em ambos os modelos, para a mesma pressuposição dos retornos à escala. A estimação do modelo de supereficiência consiste em remover a DMU em análise da restrição do modelo. Desta forma, os escores de eficiência para a DMU $i$ são:

$$
\begin{aligned}
& \text { Maximizar }_{\theta, \lambda} \phi, \\
& \text { s.a } \varphi \mathrm{y}_{\mathrm{i}}-\mathrm{Y} \lambda \leq 0, \text { exceto para } \lambda_{\mathrm{i}} \\
& \quad-\mathrm{x}_{\mathrm{i}}+\mathrm{X} \lambda \leq 0, \text { exceto para } \lambda_{\mathrm{i}} \\
& -\lambda \leq 0
\end{aligned}
$$

em que $\mathrm{Y}$ é uma matriz de $\mathrm{m}$ produtos por $\mathrm{n}$ DMUs; $X$ é uma matriz de insumos de ordem $\mathrm{k}$ insumos por $n$ DMUs; $y_{i}$ é o vetor de produtos, $x_{i}$ é o vetor de insumos, $\phi$ é um escalar e $\lambda$ é um vetor de pesos de ordem n DMUs por 1, exceto para a firma $i$. A eficiência técnica é dada por $\theta=1 / \phi$. Para a estimação do modelo sobre retornos variáveis, deve-se adicionar a restrição $N 1^{\prime} \lambda=1$, em que N1 é um vetor $n \times 1$ composto por uns.

Na escolha quanto à pressuposição dos retornos à escala, realizou-se o teste não paramétrico de duas amostras de Kolmogorov-Smirnov, sugerido por Banker (1996). O teste avalia a hipótese nula de retornos constantes de escala contra a hipótese alternativa de retornos variáveis de escala. Este teste se baseia na distância máxima vertical entre $\hat{F}^{c}\left(\ln \left(\hat{\theta}_{j}^{c}\right)\right)$ e $\hat{F}^{V}\left(\ln \left(\hat{\theta}_{j}^{v}\right)\right)$; neste caso as distribuições empíricas de $\ln \left(\hat{\theta}_{j}^{c}\right)$ e $\ln \left(\hat{\theta}_{j}^{v}\right)$, respectivamente, são usadas. A estatística toma valores entre 0 e 1 . Valores próximos de 1 tendem a rejeitar a hipótese nula e aceitar a hipótese alternativa (BANKER e NATARAJAN, 2004). 


\subsection{Regressão quantílica}

Considerando-se um grupo de variáveis tidas como exógenas e supondo-se que estas tenham influência sobre o nível de eficiência das DMUs, um modelo comumente utilizado é o Tobit (HOFF, 2007). Entretanto, tal modelo tem sido criticado na literatura de produtividade por obter coeficientes viesados (HOFF 2007; SIMAR e WILSON 2007; WILSON, 2003). Ademais, não se espera efeitos simétricos das variáveis explicativas sobre a variável dependente, pressuposição do modelo Tobit, de forma que a regressão linear sumariza o relacionamento médio entre a variável dependente e explicativa, baseado na função condicional média, permitindo apenas uma visão parcial do relacionamento (SILVA e PORTO JÚNIOR, 2006). Uma representação completa sobre tal relação, nos diferentes pontos da função de distribuição condicional, é obtida através da regressão quantílica (CAMERON e TRIVEDI, 2009), permitindo, desta maneira, distinguir diferenças na importância e na relação entre as variáveis sobre a mediana e sobre os quantis altos e baixos da variável dependente.

Por meio dos escores de eficiência obtidos pelo modelo DEA, será então operacionalizada a regressão quantílica, buscando determinar os efeitos das variáveis ambientais sobre a ineficiência técnica nos diversos pontos da distribuição condicional da variável dependente (SILVA e PORTO JÚNIOR, 2006). Isso permitirá discriminar o efeito destas sobre DMUs com medida de eficiência baixa, próximas a zero, de DMUs eficientes, escores maiores ou iguais a 1 .

A variável dependente y, representando o nível de supereficiência para cada i-ésimo município, pode ser descrita pelo modelo linear:

$$
y_{i}^{*}=x_{i}^{\prime} \beta+\varepsilon_{i}
$$

em que $x_{i}$ representa a matriz de dados (variáveis exógenas) para cada i-ésimo município, $\beta$ é matriz dos parâmetros desconhecidos e $\varepsilon_{i}$ o erro aleatório.

$$
Q_{\theta}\left(Y_{i}: X_{i}\right)=X_{i}^{\prime} \beta_{\theta} \theta \in
$$

em que $Q_{\theta}\left(Y_{i}: X_{i}\right)$ representa o $\theta$ quantil da supereficiência condicional dado o vetor de regressores. A regressão quantílica $\theta$ pode ser definida como a solução do seguinte problema:

$$
\begin{aligned}
& \min _{b \in R} n^{-1}\left\{\begin{array}{l}
\sum_{i \in\left\{i y_{i} \geq x_{\beta} \beta\right\}} \theta\left|y_{i}-x_{i} \beta\right|+ \\
\sum_{i \in\left\{i y_{y} \geq x_{\beta}\right\}}(1-\theta)\left|y_{i}-x_{i} \beta\right|
\end{array}\right\}= \\
& \min ^{-1} \sum_{i=1}^{n} \rho_{\theta}\left(y_{i}-x_{i} \beta\right)
\end{aligned}
$$

em que $\rho$ é a função "check" definida por

$$
\rho_{\theta}(u)= \begin{cases}\theta u, & u \geq 0 \\ (\theta-1) u & u<0\end{cases}
$$

em que a função $\rho_{\theta}$ multiplica os resíduos por $\theta$, se eles forem não negativos, e por $(\theta-1)$, caso contrário, para que, dessa forma, sejam tratados assimetricamente.

Para se interpretar os coeficientes da regressão quantílica, devem ser utilizadas as derivadas parciais do quantil condicional para cada regressor específico, ou seja, a mudança marginal no $\theta$-ésimo quantil condicional (HAO e NAIMAN, 2007). A mudança marginal é calculada da forma usual (CAMERON e TRIVEDI, 2009):

$$
\frac{d Q_{q}(y \mid x)}{d x_{j}}=\beta_{q j}
$$

Tal procedimento tem vários benefícios quando comparados às regressões lineares "convencionais", como parâmetros robustos a outliers e caracterização mais rica dos dados (CAMERON e TRIVEDI, 2009).

Para se avaliar a diferença estatística entre os coeficientes de cada quantil, será utilizado o teste de Wald. Sendo estimadas a variância e covariância, pode-se testar a hipótese de igualdade entre pares de coeficientes em cada quantil $\hat{\beta}_{j}^{(p)}$ e $\hat{\beta}_{j}^{(q)}$, correspondendo à mesma variância, mas entre diferentes quantis $p$ e $q$ usando a estatística de Wald (HAO e NAIMAN, 2007):

$$
\text { Estatistica }- \text { Wald }=\frac{\left(\hat{\beta}_{j}^{(p)}-\hat{\beta}_{j}^{(q)}\right)^{2}}{\hat{\sigma}_{\beta_{j}^{(p)}-\beta_{j}^{(q)}}^{(q)}}
$$

O termo $\hat{\sigma}_{\beta_{j}}^{2(p)}-\beta_{j}^{(q)}$ no denominador é a variância estimada para a diferença $\beta_{j}^{(p)}-\beta_{j}^{(q)}$, que pode ser obtida através da seguinte igualdade: 


$$
\begin{aligned}
& \operatorname{Var}\left(\hat{\beta}_{j}^{(p)}-\hat{\beta}_{j}^{(q)}\right)=\operatorname{Var}\left(\hat{\beta}_{j}^{(p)}\right)+ \\
& \operatorname{Var}\left(\hat{\boldsymbol{\beta}}_{j}^{(q)}\right)-2 \operatorname{Cov}\left(\hat{\boldsymbol{\beta}}_{j}^{(p)}, \hat{\boldsymbol{\beta}}_{j}^{(q)}\right)
\end{aligned}
$$

Sobre a hipótese nula, a estatística de Wald segue distribuição $\chi^{2}$ com $q$ graus de liberdade, sendo $q$ o número de hipóteses testadas conjuntamente ou distribuição $F$, sendo:

$$
F=\frac{1}{q} W
$$

Com q graus de liberdade no numerador e graus de liberdade no denominador (CAMERON e TRIVEDI, 2009).

\subsection{Fonte e tratamento dos dados}

\subsubsection{Análise envoltória de dados}

Quanto às variáveis a serem utilizadas, têm sido desenvolvidos, na literatura de produtividade, procedimentos objetivos para a seleção de variáveis, visto que, quando um grande número de variáveis é empregado, há uma tendência de as firmas serem consideradas eficientes, reduzindo o poder de discriminação do modelo (CUNHA et al., 2006; FERREIRA e GOMES, 2009). Entretanto, a relação tamanho da amostra/número de variáveis utilizadas assume o valor pequeno, não necessitando da utilização de procedimentos para selecioná-las (LINS e MOREIRA, 2000). De qualquer forma, as variáveis utilizadas representam a maior parcela dos insumos, compreendendo $70 \%$ dos custos despendidos em insumos. Os insumos foram agrupados em terra, capital e trabalho, sendo os dois últimos medidos em termos de fluxo. Já para o insumo terra, dada a não disponibilidade de dados sobre o valor de aluguel das terras próprias, utilizou-se a área das propriedades, como proxy do valor das terras.

$\mathrm{Na}$ estimação da eficiência técnica ${ }^{7}$ dos modelos CCR, BCC e supereficiência sobre a pressuposição de retornos constantes e variáveis dos

\footnotetext{
7 Na estimação do modelo DEA, os municípios de Raposos e Santa Cruz de Minas foram retirados da amostra, visto que o Censo Agropecuário identifica com o caractere " $\mathrm{X}$ " dados que possuem menos de 3 (três) informantes. Características assim identificadas foram consideradas iguais a zero.
}

municípios mineiros (853), foram utilizadas as variáveis abaixo obtidas a partir do Censo Agropecuário 2006:

Receita agropecuária dos municípios em $R$ \$ 1.000: obtida por meio da soma das receitas da produção agropecuária;

\section{Insumos:}

Terra: área total dos estabelecimentos agropecuários em ha; Mão de Obra: valor obtido pelo somatório dos salários pagos à mão de obra familiar e à contratada, em R\$1.000; Defensivos: valor despendido em R\$ 1.000 com agrotóxicos, adubos, corretivos, sementes e mudas; Animais: valor despendido em R\$ 1.000 com medicamentos para animais, sal, rações industrializadas e compra de animais; Serviços: valor despendido em R\$ 1.000 com aluguel de máquinas e equipamentos, transporte da produção e serviços de empreitada; Financeiras: valor despendido em R\$ 1.000 com juros e despesas financeiras, impostos e taxas e Energia: valor despendido em R\$ 1.000 em energia elétrica e combustíveis.

\subsubsection{Regressão quantílica}

Na estimação da regressão quantílica foi utilizado o pacote estatístico Stata versão 10.1, sendo os parâmetros estimados por métodos de programação linear (simplex). As variáveis foram obtidas a partir do Censo Agropecuário 2006 para todos os municípios mineiros.

- Dependente: y - Nível de supereficiência dos municípios.

- Explicativas: Plantio em nível: Área total dos estabelecimentos agrícolas que plantam em nível em ha/área total dos estabelecimentos em ha; Terraços: Área total dos estabelecimentos agrícolas que utilizam terraços em ha/área total dos estabelecimentos agrícolas em ha; Rotação: Área total dos estabelecimentos cultivadas em sistema de rotação de culturas em ha/ área total dos estabelecimentos em ha; Renovação: Área total dos estabelecimentos utilizada com lavouras para reforma e/ou renovação e/ou recuperação de pastagens 
Tabela 1. Estimativas e estatísticas dos escores de eficiência.

\begin{tabular}{cc}
\hline Estatísticas Descritivas & \\
\hline Número de Observações & 851 \\
Média & 0,1896 \\
Desvio Padrão & 0,3194 \\
Valor Mínimo & 0,0179 \\
Valor Máximo & 1 \\
\hline
\end{tabular}

Obs: As eficiências para cada município podem ser obtidas com os autores.

em ha/área total dos estabelecimentos em ha; Pousio: Área total dos estabelecimentos utilizada em pousio ou descanso de solos em ha/área total dos estabelecimentos em ha; Queimadas: Área total dos estabelecimentos não queimada em ha/área total dos estabelecimentos em ha; Proteção: Área dos estabelecimentos em que foram utilizadas práticas de conservação e/ou proteção de encostas em ha/área total dos estabelecimentos em ha; Matas: Área dos estabelecimentos agrícolas com matas e/ou florestas naturais destinadas à preservação permanente ou reserva legal em ha/área total dos estabelecimentos em ha; Matas2: Área dos estabelecimentos agrícolas com matas e/ou florestas naturais (excluindo-se a área de preservação permanente e as em sistemas agroflorestais) em ha/área total dos estabelecimentos em ha; Degradadas: Área dos estabelecimentos agrícolas não erodidas, degradadas, salinizadas etc. em ha/área total dos estabelecimentos em ha; Hídrico: Número de estabelecimentos agropecuários com recursos hídricos em ha/número total dos estabelecimentos agrícolas.

\section{Resultados}

\subsection{Estimação da eficiência técnica}

Após a estimação dos níveis de eficiência técnica sobre a pressuposição de retornos constantes e retornos variáveis ${ }^{8}$ realizou-se o teste de

${ }_{8}$ Os escores de supereficiência dos municípios de Divisa Alegre, Jenipapo de Minas, Nova Lima, Ribeirão Vermelho e Sapucaí-Mirim não são tratados ao longo do artigo e
Kolmogorov-Smirnov ${ }^{9}$ de duas amostras, tanto para os modelos "convencionais" quanto para o modelo de supereficiência. Através deste teste, os valores de 0,674912 e 0,183428 foram obtidos, respectivamente, para o modelo que restringe a eficiência a 1 e para o modelo de supereficiência, ambos significativos a $1 \%$, indicando que devem ser utilizados os escores obtidos do modelo com pressuposição de retornos variáveis à escala (VRS). Tal resultado era esperado visto que o modelo CCR pressupõe escala ótima para as firmas; entretanto, competição imperfeita, restrições financeiras etc. levam às DMUs a operar fora dessa escala.

A eficiência obtida pelo método "tradicional" será interpretada neste tópico, mas, considerando-se que $11 \%$ dos municípios obtiveram escores de eficiência iguais a 1 , o que pode comprometer as estimativas na regressão quantílica, foram estimados "novos" escores para cada município pelo modelo com supereficiência. Estes "novos" escores são utilizados exclusivamente na regressão quantílica. O método de estimação com supereficiência permite que os escores dos municípios eficientes variem de zero a infinito, enquanto os escores dos municípios ineficientes não variam, permitindo melhores resultados na regressão quantílica.

Pelas estimativas sobre a pressuposição de retornos variáveis, a média da eficiência para Minas Gerais foi de apenas 18,96\% (Tabela 1), ou

também não foram incluídos na estimação do modelo de regressão quantílica pelo fato de seus escores retornarem "big". Este resultado é obtido quando a DMU em análise não pode ser avaliada, uma vez que há uma distância enorme entre o ponto referente a esta DMU na fronteira de possibilidades de produção e o ponto supereficiente.

9 O teste de duas amostras de Kolmogorov-Smirnov foi implementado através do software StatsDirect versão 2.7.2. 
seja, a produção poderia aumentar, em média, $81,04 \%$ com a correção no nível de utilização dos insumos. As estatísticas da Tabela 1 mostram a amplitude dos valores de eficiência estimados variando de 0,0178974 a 1 .

A seguir, o histograma das medidas de eficiência técnica com pressuposição de retornos variáveis à escala é apresentado, permitindo uma apresentação da distribuição dos níveis de eficiência. Pela Figura 1, os níveis de eficiência se concentram abaixo de 0,2 , com $11 \%$ das firmas apresentando eficiência igual a 1 (DMUs eficientes).

A Tabela 2 compara os níveis de produto e insumos utilizados. Apesar das DMUs eficientes utilizarem mais insumos, o nível de produto é proporcionalmente maior. As firmas eficientes produzem $63 \%$ a mais que as ineficientes, e na média utilizam $46 \%$ mais insumos. Desta forma, os municípios considerados ineficientes podem aumentar proporcionalmente a produção, ao nível das firmas eficientes, mas não o produzem provavelmente devido a falhas e dificuldades de gestão da propriedade rural.

\subsection{Regressão quantílica}

A partir dos escores de supereficiência para cada município, estimou-se a regressão quantílica no segundo estágio, analisando a relação entre os escores e as variáveis ambientais descritas anteriormente para os quantis 0,$10 ; 0,25 ; 0,50 ; 0,75$; e 0,90 . As estimativas são apresentadas na Tabela 3, junto ao desvio padrão entre parênteses para cada coeficiente. As variáveis "Rotação", "Queimadas"

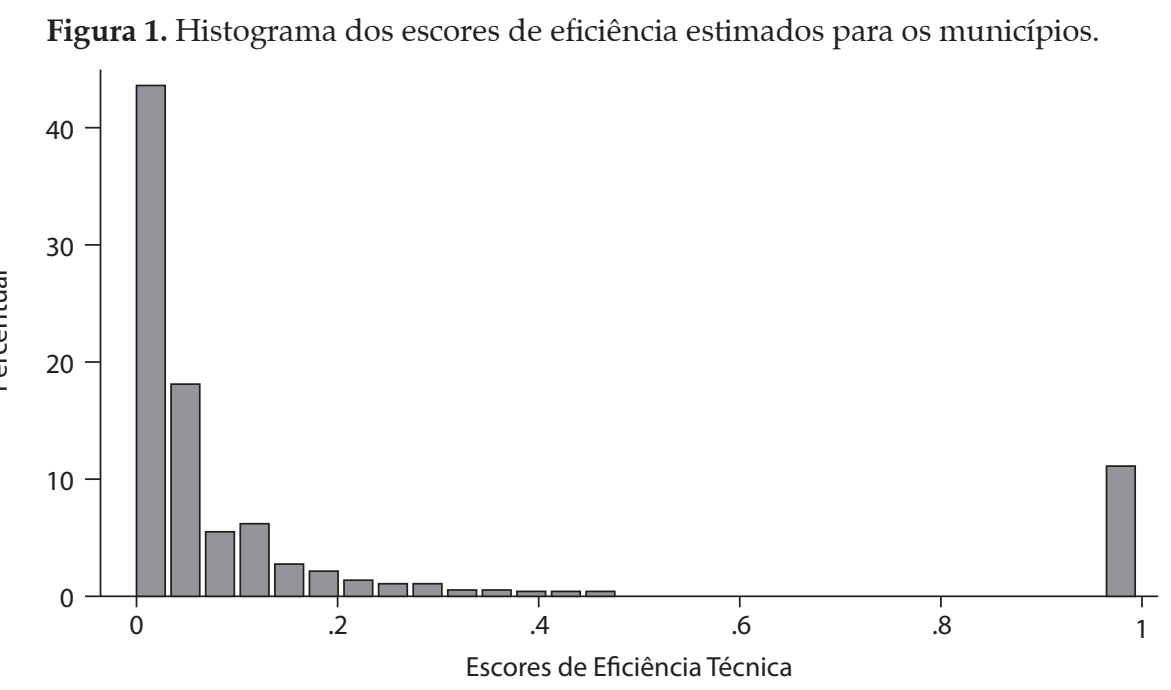

Tabela 2. Comparação entre os municípios eficientes e ineficientes quanto à receita e insumos.

\begin{tabular}{lcccc}
\hline \multirow{2}{*}{ Especificação } & \multirow{2}{*}{ Dimensão } & \multicolumn{2}{c}{ Grupos de Municípios } & \multirow{2}{*}{ Diferença (\%) } \\
\cline { 3 - 4 } & & Ineficientes & Eficientes & \\
\hline Receitas & $\mathrm{R} \$$ & $13.534,69$ & $36.602,10$ & $63 \%$ \\
Terra (ha) & hectares & $35.338,78$ & $62.153,79$ & $43 \%$ \\
Mão de Obra & $\mathrm{R} \$$ & $4.101,93$ & $5.911,60$ & $31 \%$ \\
Defensivos & $\mathrm{R} \$$ & $4.090,40$ & $11.816,98$ & $65 \%$ \\
Animais & $\mathrm{R} \$$ & $2.718,33$ & $5.603,09$ & $51 \%$ \\
Serviços & $\mathrm{R} \$$ & $1.447,65$ & $3.918,18$ & $63 \%$ \\
Financeiro & $\mathrm{R} \$$ & 307,45 & 899,84 & $66 \%$ \\
Energia & $\mathrm{R} \$$ & $2.896,01$ & $3.035,35$ & $5 \%$ \\
\hline
\end{tabular}


Tabela 3. Estimativas dos modelos da regressão quantílica.

\begin{tabular}{|c|c|c|c|c|c|}
\hline Parâmetros/Quantis & q 0.10 & q 0.25 & q 0.50 & q 0.75 & q 0.90 \\
\hline \multirow{2}{*}{ Plantio em Nível } & $0.051^{* * *}$ & $0.050^{* * *}$ & $0.053^{* *}$ & 0.063 & -0.074 \\
\hline & $(0.016)$ & $(0.018)$ & $(0.024)$ & $(0.058)$ & $(0.132)$ \\
\hline \multirow{2}{*}{ Terraços } & 0.003 & 0.071 & 0.261 & $1.071^{* * *}$ & $1.489 * *$ \\
\hline & $(0.036)$ & $(0.072)$ & $(0.169)$ & $(0.272)$ & $(0.705)$ \\
\hline \multirow{2}{*}{ Renovação } & -0.027 & $-0.051^{*}$ & $-0.101^{* *}$ & $-0.221^{* * *}$ & $-0.571^{* * *}$ \\
\hline & $(0.027)$ & $(0.030)$ & $(0.045)$ & $(0.074)$ & $(0.143)$ \\
\hline \multirow{2}{*}{ Pousio } & -0.042 & -0.020 & $-0.116^{* * *}$ & $-0.307^{* * *}$ & $-0.400^{*}$ \\
\hline & $(0.038)$ & $(0.040)$ & $(0.046)$ & (0.105) & $(0.225)$ \\
\hline \multirow{2}{*}{ Matas } & $-0.237^{* * *}$ & $-0.323^{* * *}$ & $-0.352^{* * *}$ & $-0.641^{* * *}$ & $-1.027^{* * *}$ \\
\hline & $(0.060)$ & $(0.059)$ & $(0.084)$ & (0.203) & $(0.351)$ \\
\hline \multirow{2}{*}{ Matas2 } & $-0.138^{* *}$ & $-0.244^{* * *}$ & $-0.380^{* * *}$ & $-0.559^{* * *}$ & $-1.512^{* * *}$ \\
\hline & $(0.035)$ & $(0.044)$ & $(0.084)$ & $(0.145)$ & $(0.503)$ \\
\hline \multirow{2}{*}{ Degradadas } & 0.042 & 0.589 & 0.145 & -2.140 & -5.976 \\
\hline & $(0.475)$ & $(0.713)$ & $(1.559)$ & $(3.207)$ & $(5.056)$ \\
\hline \multirow{2}{*}{ Hídrico } & -0.020 & -0.0260 & $-0.091^{* *}$ & -0.093 & $-0.585^{* *}$ \\
\hline & $(0.021)$ & $(0.025)$ & $(0.039)$ & $(0.089)$ & $(0.295)$ \\
\hline \multirow{2}{*}{ Constante } & 0.081 & -0.404 & 0.169 & 2.609 & 7.292 \\
\hline & $(0.476)$ & $(0.715)$ & (1.553) & (3.219) & $(5.047)$ \\
\hline
\end{tabular}

* Significativo a $10 \% ; * *$ Significativo a $5 \%$ e *** Significativo a $1 \%$.

e "Proteção" foram excluídas do modelo por não serem estatisticamente significativas isoladamente, pelo teste $\mathrm{t}$, e conjuntamente pelo teste $\mathrm{F}$, ao nível de significância de $1 \%$ nos diversos quantis estimados, em que o maior valor obtido para o teste $\mathrm{F}_{(3,838)}=1,40$ no quantil 0,25 . Tal modelo permite não somente avaliar a relação entre variáveis ambientais e a eficiência produtiva dos diversos municípios mineiros, mas também determinar a variação da importância destas variáveis sobre os municípios de acordo com os níveis de ineficiência da produção agropecuária (mais eficientes e menos eficientes).

Inicialmente, esperava-se que as variáveis explicativas apresentassem sinais positivos, de forma que um aumento na utilização das práticas agrícolas e melhorias na qualidade dos recursos e na disponibilidade dos recursos hídricos aumentasse a eficiência técnica. De outra forma, permitiria o aumento da produção, dado nível de utilização dos insumos, pois o aumento na utilização das práticas agrícolas melhoraria as condições edafoclimáticas dos estabelecimentos, contribuindo para o aumento da produção. Por exemplo, com a utilização do plantio em nível, espera-se que os solos não sejam degradados, ou pelo menos tal degradação seja reduzida, o que permite reduzir as perdas de nutrientes, aumentar a absorção destes pelas plantas e, assim, aumentar a produtividade.

Entretanto, a relação entre as variáveis e a eficiência se mostrou diferente do esperado, como para "Plantio em Nível", "Hídrico" e "Degradadas", apresentando, para pelo menos um quantil, relação negativa com a eficiência. A variável "Renovação" também apresentou sinal negativo não esperado; entretanto, este resultado pode ser atribuído à baixa produtividade da produção pecuária extensiva, já que esta variável refere-se à recuperação de áreas com pastagem.

As estimativas obtidas indicam que a importância das variáveis ambientais aumenta à medida que a eficiência técnica também se eleva. Tal comportamento era esperado, pois considera-se que as DMUs ineficientes têm como fator de importância a baixa capacidade de gestão da produção, enfrentando dificuldades até mesmo para implantar tais práticas agrícolas adequadamente. O aumento da ineficiência reduz a eficácia da uti- 
lização do plantio em nível, de forma que, quanto maior a primeira, menores os efeitos da segunda.

Todas as variáveis incluídas no modelo apresentaram o comportamento esperado quanto ao aumento de importância sobre a eficiência, à medida que esta aumenta, em valor absoluto. De acordo com a Tabela 3, as variáveis com maior importância sobre a eficiência foram, respectivamente, "Degradadas", "Matas2", "Terraços" e "Matas". Calculando seus efeitos marginais por meio da equação (6), obtém-se que o aumento em um ponto percentual com plantio utilizando terraços aumenta a eficiência em 1,071 ponto percentual para o quantil 0,75 , acrescendo sua importância para 1,489 no quantil 0,90. Este resultado demonstra a importância da utilização de terraços no estado de Minas Gerais, provavelmente pela topografia mineira, caracterizada por apresentar relevo acidentado, principalmente nas regiões mais ao sul do estado e que também têm o maior número de municípios, onde a utilização destas possui um efeito expressivo na manutenção da qualidade dos solos (estrutura e composição química e orgânica), o que permite que culturas agrícolas tenham maior produtividade.

A variável "Matas2", que trata da proporção dos estabelecimentos com matas e/ou florestas naturais excluindo-se áreas de preservação per- manente e em sistemas agroflorestais, apresentou efeito negativo sobre a eficiência. Um aumento em um ponto percentual na área destinada às matas reduz a eficiência em 0,$237 ; 0,323 ; 0,352 ; 0,641$ e 1,027 pontos percentuais para os quantis 0,10 ; 0,$25 ; 0,50 ; 0,75$; e 0,90 , respectivamente. Este efeito pode ser interpretado como resultado da redução da área destinada à atividade agropecuária. Como considerado na estimação da eficiência, os insumos agropecuários são, de forma geral, imóveis e, desta forma, os insumos seriam "subutilizados" para um mesmo nível produtivo, o que contribuiria para o aumento da ineficiência (a quantidade utilizada dos insumos permanece constante, mas a produção se reduz). A variável "Matas" e "Pousio" apresentaram comportamento semelhante, que pode ser interpretado tal como "Matas2".

Como a regressão quantílica pressupõe que as variáveis têm efeitos explicativos diferenciados entre os diversos quantis da variável dependente, torna-se importante a verificação desta diferença estatisticamente. Desta forma, a Tabela 4 apresenta o resultado do teste de Wald para os diversos parâmetros entre os quantis.

Pela Tabela 4, apenas as variáveis "Terraços", "Renovação", "Pousio" e "Matas2" são estatisticamente diferentes entre os quantis estimados: estas variáveis aumentam, estatisticamente, sua

Tabela 4. Testes de hipótese do modelo estimado.

\begin{tabular}{|c|c|c|c|}
\hline Hipótese Nula & $\mathbf{F}_{\text {calc }}$ & $\mathbf{F}_{\text {Crít }(4,841) \mathrm{gl}}$ & Decisão \\
\hline $\begin{array}{l}\text { Ho: Igualdade do Parâmetro "Plantio em Nível" } \\
\text { entre todos os quantis estimados }\end{array}$ & 0,47 & $1,951555^{*}$ & Não se rejeita Ho \\
\hline $\begin{array}{l}\text { Ho: Igualdade do Parâmetro "Terraços" } \\
\text { entre todos os quantis estimados }\end{array}$ & 4,25 & $3,341517^{* * *}$ & Rejeita-se Ho \\
\hline $\begin{array}{l}\text { Ho: Igualdade do Parâmetro "Renovação" } \\
\text { entre todos os quantis estimados }\end{array}$ & 3,81 & $3,341517^{* * *}$ & Rejeita-se Ho \\
\hline $\begin{array}{l}\text { Ho: Igualdade do Parâmetro "Pousio" } \\
\text { entre todos os quantis estimados }\end{array}$ & 2,43 & $3,341517^{* * *}$ & Rejeita-se Ho \\
\hline $\begin{array}{l}\text { Ho: Igualdade do Parâmetro "Matas" } \\
\text { entre todos os quantis estimados }\end{array}$ & 1,90 & $1,951555^{*}$ & Não se Rejeita Ho \\
\hline $\begin{array}{l}\text { Ho: Igualdade do Parâmetro "Matas2" } \\
\text { entre todos os quantis estimados }\end{array}$ & 4,30 & $3,341517^{* * *}$ & Rejeita-se Ho \\
\hline $\begin{array}{l}\text { Ho: Igualdade do Parâmetro "Degradadas" } \\
\text { entre todos os quantis estimados }\end{array}$ & 0,63 & $1,951555^{*}$ & Não se Rejeita Ho \\
\hline $\begin{array}{l}\text { Ho: Igualdade do Parâmetro "Hídrico" } \\
\text { entre todos os quantis estimados }\end{array}$ & 1,50 & $1.951555^{*}$ & Não se Rejeita Ho \\
\hline
\end{tabular}


importância quanto maior a eficiência, ao passo que as demais possuem igual influência sobre a eficiência, seja maior ou menor.

Desta forma, apenas as variáveis mais importantes de fato apresentam efeitos diferenciados para os diversos níveis de ineficiência dos municípios mineiros. Este resultado, entretanto, não inviabiliza o modelo, pois a estimação por Mínimos Quadrados Ordinários ou qualquer outro que estimaria a relação entre as variáveis explicativas e a ineficiência média não captaria os efeitos diferenciados deste modelo para estas variáveis.

\section{Conclusões}

As estimativas para a eficiência produtiva dos municípios mineiros apresentam grande amplitude entre máximos e mínimos, embora com escores baixos, de maneira geral, o que demonstra a necessidade de medidas de correção na forma como os insumos agropecuários estão sendo utilizados pelos estabelecimentos agropecuários, o que eleva os custos unitários e reduz a competitividade da produção agropecuária do estado.

O estudo avaliou a relação entre variáveis ambientais e de ação antrópica sobre o meio ambiente, demonstrando que as variáveis de maior importância referem-se àquelas relacionadas à qualidade/conservação do solo e presença de matas. A importância da utilização dos terraços pelos estabelecimentos pode ser atribuída ao relevo acidentado da parte mais ao sul de Minas Gerais, sendo esta área também a que tem o maior número de municípios. Em relação à presença de matas, não se encontrou a relação esperada com a eficiência dos municípios, pois houve redução na eficiência quando suas áreas foram aumentadas. Esse comportamento pode ser atribuído à redução proporcional da área agropecuária quando comparada ao nível de utilização dos insumos.

Uma limitação na estimação da eficiência produtiva agropecuária refere-se ao nível de agregação dos dados que não permitem estimar tal medida de desempenho para cada produtor e por atividade produtiva, o que provocaria maior interesse. Entretanto, os resultados obtidos for- necem uma proxy satisfatória do desempenho produtivo, uma vez que tal medida apresentou-se baixa, permitindo concluir que grande parte dos produtores são ineficientes. Como sugestão para estudos posteriores, sugere-se o estudo de áreas com características topográficas semelhantes, buscando avaliar se de fato tal resultado se deve à uma distorção das estimativas.

\section{Referências bibliográficas}

ALVIM, M. I. S. A. e OLIVEIRA JUNIOR, L. B. Análise da competitividade da produção de soja no sistema de plantio direto no Estado de Mato Grosso do Sul. Revista de Economia e Sociologia Rural, v. 43, n. 3, p. 505-528, 2005.

ANDERSEN, P. e PETERSEN, N. C. A procedure for ranking efficient units in Data Envelopment Analysis. Management Science, v. 39, n. 10, p. 1261-1264, 1993.

ARAÚJO, P. M. Q. e CARMONA, C. U. M. Eficiência de uma rede de agências bancárias utilizando o modelo Data Envelopment Analysis (DEA). Revista da Associação Brasileira de Engenharia de Produção. Florianópolis - SC:, v. 2, n. 2, p. 1-25, 2002.

AZAMBUjA, A. M. V. Análise de eficiência na gestão do transporte urbano por ônibus em municípios brasileiro. $385 f$. Tese (Doutorado) - Universidade Federal de Santa Catarina, Florianópolis, 2002.

BACEN - BANCO CENTRAL DO BRASIL. Investimento Estrangeiro Direto - 2008. 2008. Disponível em <http:// www.bcb.gov.br/rex/IED/Port/ingressos/htms/index3. asp?idpai=invedir $>$. Acesso em 20 jan. 2010.

BANKER, R. D. Hypothesis tests using data envelopment analysis. The Journal of Productivity Analysis, v. 7, n. 2-3, p. 139-159, 1996.

BANKER, R. D.; CHARNES, A. e COOPER, W. W. Some models for estimating technical and scale inefficiencies in data envelopment analysis. Management Science, v. 30, n. 9, p. 1078-1092, 1984.

BANKER, R. D. e NATARAJAN, R. Statistical tests based on DEA efficiency scores. In: COOPER, W.; SEIFORD, L. e ZHU, J. Handbook on Data Envelopment Analysis. Kluwer Academic Publishers, Inc., New York, Chapter. v. 11, p. 265-298, 2004.

BATALHA, M. O. e SCARPELLI, M. Gestão do Agronegócio: aspectos conceituais. In: BATALHA, M. O. Gestão do Agronegócio: textos selecionados. São Carlos, UFSCAR, 2005. 
BOLES, J. Efficiency squared: Efficiency computations of efficiency indexes. Western Farm Economics Association Proceedings, p. 137-142, 1966.

BRASIL. MINISTÉRIO DA AGRICULTURA, PECUÁRIA E ABASTECIMENTO. Intercâmbio comercial do agronegócio: Principais mercados de destino. Brasília: Ministério da Agricultura, Pecuária e Abastecimento/ Assessoria de Comunicação Social, 2009, 469 p.

BURGESS, J. F. e WILSON, P. W. Technical efficiency in veterans administration hospitals. In: FRIED, H. O.; LOVELL, C. A. K. e SCHMIDT, S. S. The measurement of productive efficiency: Techniques and applications. New York: Oxford, p. 335-351, 1993.

CAMERON, A. C. e TRIVEDI, P. K. Microeconomics using Stata. Stata Press Publications, College Station, Texas, p. 692, 2009.

CARNEIRO, P. A. S.; FONTES, M. P. F. e FONTES, R. Índice pedológico e de Manejo de Solos como Suporte ao Estudo das Disparidades Microrregionais em Minas Gerais. In: FONTES, R. e FONTES, M. P. F. Crescimento e Desigualdade Regional em Minas Gerais. Viçosa: Editora: Folha de Viçosa, p. 249-292, 2005.

CEPEA - CENTRO DE ESTUDOS AVANÇADOS EM ECONOMIA APLICADA. 2009. 16p. Agronegócio mineiro: Segundo semestre pode ser melhor. Disponível em < www.agricultura.mg.gov.br/info/relatorio_pib/ pib_out_2009.pdf > . Acesso em 18 dez. 2009.

CESPEDES, J. C. e LEANDRO, R. A. Eficiência técnica de fazendas produtoras de leite: uma abordagem bayesiana. In: SEAGRO, 2005, Londrina, Anais... Londrina: Editora, 2005.

CHARNES, A.; COOPER, W. W. e RHODES, E. Measuring the efficiency of decision making units. European Journal of Operations Research, v. 2, n. 6, p. 429-444, 1978.

CHILINGERIAN, J. A. Evaluating physician efficiency in hospitals: A multivariate analysis of best practices. European Journal of Operational Research, v. 80, n. 00, p. 548-574, 1995.

COELLI, T. J. A guide to FRONTIER version 4.1: A computer program for stochastic frontier production and cost function estimation. CEPA Working Paper 96/07. Department of Economics, University of New England, Armidale, Australia, 1999.

CUNHA, B. T; SOARES DE MELLO, J. C. C. B. e ANGULO-MEZA, L. Implementação Computacional de Seleção de Variáveis em DEA: um Estudo de Caso em Avaliação Educacional. In: CLAIO - Congreso LatinoIberoamericano de Investigación Operativa,13, 2006, Montevideu. Anais... XIII CLAIO Congreso LatinoIberoamericano de Investigación Operativa, 2006.
DEBREU, G. The measurement of productive efficiency. Econometrica, v. 19, n. 3, p. 273-292, 1951.

EL KHALILI, A. Commodities ambientais em missão de paz - novo modelo econômico para a América Latina e o Caribe. São Paulo, SP: Nova Consciência, p. 271, 2009.

ENGSTRÖM, R. et. al. Environmental assessment of Swedish agriculture. Ecological Economics, v. 60, n. 00, p. 550-563, 2007.

FERREIRA, A. H. Eficiência de sistemas de produção de leite: uma aplicação da análise envoltória de dados na tomada de decisão. 2002. 120 f. Dissertação (Mestrado) Universidade Federal de Viçosa, Viçosa.

FERREIRA C. M. C. e GOMES, A. P. Introdução à análise envoltória de dados: Teoria, modelos e aplicações. Viçosa, MG: Editora UFV, p. 389, 2009.

FETHI, M. D. Measuring the efficiency of european airlines: An application of DEA and Tobit analysis. In: Annual Meeting of the European Public Choice Society, Siena, Italy, 26-29 April, 2000, European Public Choice Society, Siena.

GOMES, A. P. Impactos das transformações da produção de leite no número de produtores e requerimentos de mão de obra e capital. 1999. 161f. Tese (Doutorado) - Universidade Federal de Viçosa, Viçosa.

GOMES, A. P. e BAPTISTA, A. J. M. S. Análise envoltória de dados: conceitos e modelos básicos. In: SANTOS, L. M. e VIEIRA, W. C. (Ed.). Métodos quantitativos em economia. Viçosa: Editora UFV, p.121-160, 2004.

GONÇALVES, R. M. L. et al. Analysis of technical efficiency of milk-producing farms in Minas Gerais. Revista de Economia Aplicada, São Paulo, SP, v. 12, n. 2, p. 321-335, 2008.

GREEN, R. H.; DOYLEA, J. R. e COOK, W.D. Preference voting and project ranking using DEA and crossevaluation. European Journal of Operational Research, v. 90, n. 3, p. 461-472, 1996.

HASHIMOTO, A. A ranked voting system using a DEA/AR exclusion model: A note. European Journal of Operational Research, v. 97, n. 3, p. 600-604, 1997.

HAO, L. e NAIMAN, D. Q. Quantile regression. Sage Publications, Inc. p. 125. 2007.

HOFF, A. Second stage DEA: Comparison of approaches for modeling the DEA score. European Journal of Operational Research, v. 181, n. 1, p. 425-435, 2007.

KUMBHAKAR, S. C. e LOVELL, C. A. K. Stochastic Frontier Analysis. United Kingdom: Cambridge University Press, p. 333, 2000.

KOOPMANS T. Activity analysis of production and allocation. John Wiley \& Sons, New York. 1951. 
LEE, H. Y. e PARK, Y. T. An International Comparison of R\&D Efficiency: DEA Approach. Asian Journal of Technology Innovation, v. 13, n. 2, p. 207-222, 2005.

LINS, M. P. S. e MEZA, L. A. Modelos DEA clássicos. In: LINS, M. P. S. e MEZA, L. A. Análise envoltória de dados e perspectivas de integração no ambiente do apoio à decisão. Rio de Janeiro: COPPE/UFRJ, p. 7-36, 2000.

LINS, M. P. E. e MOREIRA, M. C. B. Implementação com seleção de variáveis em DEA. In: LINS, M. P. S. e MEZA, L. A. Análise envoltória de dados e perspectivas de integração no ambiente do apoio à decisão. Rio de Janeiro: COPPE/UFRJ, p. 37-52, 2000.

LOPÉZ, R. Environmental externalities in traditional agriculture and the impact of trade liberalization: the case of Ghana. Journal of Development Economics, v. 53, n. 1, p. 17-39, 1997.

MOITA, M. H. V. Medindo a eficiência relativa de escolas municipais da cidade do Rio Grande do Sul - RS usando a abordagem DEA (Data Envelopment Analysis). 1995. 105f. Dissertação (Doutorado) - Universidade Federal de Santa Catarina, Florianópolis.

MOREIRA, A. J. B. Estimativa regional da produtividade agropecuária. In: CONGRESSO DA SOCIEDADE BRASILEIRA DE ECONOMIA E SOCIOLOGIA RURAL, 15, 2002, Passo Fundo. Anais... Brasília: SOBER, 2002.

PASCUAL, R. F. Eficiência de los centros públicos de educación secundaria de La provincia de Alicante. Alicante, Espanha: Universidad de Alicant, 2000. 237f. Tese (Douctorado em Ciencias Económicas) - Universidad de Alicante, Alicante.

RAY, S. C. e KIM, H. J. Cost efficiency in the US steel industry: A nonparametric analysis using data envelopment analysis. In: European Journal of Operational Research, v. 80, n. 00, p. 654-671, 1995.

RODRIGUES, W. Valoração Econômica dos Impactos Ambientais de Tecnologias de Plantio em Região de
Cerrados. Revista de Economia e Sociologia Rural, Rio de Janeiro, v. 43, n. 01, p. 135-153, 2005.

RUGGIERO, J. e VITALIANO, D. F. Assessing the efficiency of public schools using data envelopment analysis and frontier regression. Contemporary Economic Policy, v. 17, n. 3, p. 32 1-331, 1999.

SIDRA - SISTEMA IBGE DE RECUPERAÇÃO AUTOMÁTICA. Produção Agrícola Municipal - 2008. Disponível em < http://www.sidra.ibge.gov.br/bda/ pesquisas/pam/>. Acesso em: 7 jan 2010.

SILVA, E. N. e PORTO JÚNIOR, S. S. Sistema Financeiro e Crescimento Econômico: Uma Aplicação de Regressão Quantílica. Economia Aplicada, v. 10, n.3, p. 425-442, 2006.

SIMAR, L. e WILSON, P. W. Estimation and inference in two-stage, semi-parametric models of production processes. Journal of Econometrics, v. 136, n. 1, p.31-64, 2007.

SOARES, W. L; FREITAS, E. A. V. e COUTINHO, J. A. G. Trabalho rural e saúde: intoxicação por agrotóxicos no município de Teresópolis-RJ. Revista de Economia e Sociologia Rural, v. 43, n. 4, p. 685-701, 2005.

WILSON, P. W. Testing independence in models of produtive efficiency. Journal of Productivity Analysis, v. 20, n. 3, p. 361-390, 2003.

ZILBERMAN, D. et. al. Agriculture and the environment: an economic perspective with implications for nutrition. Food Policy, v. 24, n. 2-3, p. 211-229, 1999.

ZUCCOLOTTO, R.; ABRANTES, L. A.; JÚNIO, A. C. B. e GOMES, A. P. Impacto do sistema tributário mineiro na cadeia produtiva do leite. In: Congresso Internacional do Leite - Fórum das Américas: Leite e Derivados, 7, 2009, Juiz de Fora. Anais... Juiz de Fora: Embrapa Gado de Leite, p. 203-237, 2009. 